\title{
Akute myeloische Leukämie mit pulmonaler Manifestation
}

\author{
E. Schalk \\ A. Franke \\ M. Koenigsmann
}

Acute Myeloid Leukemia with Pulmonary Manifestation

\section{Zusammenfassung}

Hintergrund: Extramedulläre Manifestationen von Leukämien werden oft fehlgedeutet. Die Prognose von pulmonal manifestierten Leukämien ist schlecht. Kasuistik: Eine 57-jährige Patientin wurde wegen einer therapierefraktären Pneumonie sowie des Verdachts auf eine akute Leukämie in unsere Klinik verlegt. Anhand des Knochenmarkes wurde eine akute myeloische Leukämie (AML, FAB M5a) gesichert. Radiologisch fielen ausgedehnte Verschattungen beider Lungen auf. In einer bronchoalveolären Lavage dominierten leukämische Blasten, so dass die Diagnose einer AML mit pulmonaler Manifestation gestellt wurde. Die Leukämietherapie mit Cytarabin/Idarubicin erfolgte unter zusätzlicher Gabe von Prednisolon. Im Verlauf verbesserte sich der pulmonale Befund sowohl klinisch als auch radiologisch. Nach der Induktionstherapie konnte eine komplette hämatologische Remission (CR) gesichert werden. Zwei Konsolidierungstherapien folgten. Die Patientin verstarb vier Monate nach Erreichen der CR. Schlussfolgerungen: Die pulmonale Manifestation einer AML wird bronchoskopisch gesichert. Der initial günstige Verlauf dieses Falles ist möglicherweise auf den Zusatz von Prednisolon zur Chemotherapie zurückzuführen.

\section{Abstract}

Background: Extramedullary manifestations of leukemias are often misinterpreted. The prognosis of pulmonary manifestated leukemias is poor. Case report: A 57-year-old female patient was admitted to our hospital because of refractory pneumonia and the suspicion of acute leukemia. The diagnosis of acute myeloid leukemia (AML, FAB M5a) was derived from bone marrow aspiration. The radiograph and the computed tomography of the chest showed infiltrations of both lungs. The cytologic aspect of a bronchoalveolar lavage was dominated by leukemic blasts, therefore AML with pulmonary manifestation was diagnosed. Cytotoxic therapy with cytarabine/idarubicin was combined with prednisolone. In the course of treatment the pulmonary function improved and radiographic alterations diminished significantly. After induction therapy complete haematological remission (CR) was shown by bone marrow aspiration. Two consolidation therapies followed. Unfortunately, the patient died four months after CR in a local hospital. Conclusions: The pulmonary manifestation of AML can be diagnosed by bronchoscopy. In contrast to the literature the initial course of the disease in this case was favourable possibly due to the addition of prednisolone to the cytotoxic treatment.

Institutsangaben

Klinik für Hämatologie/Onkologie, Otto-von-Guericke-Universität Magdeburg

(Direktor: Frau Prof. Dr. med. A. Franke)

Anmerkung

Der Inhalt dieser Arbeit wurde bereits auf der Herbsttagung der Ostdeutschen Studiengruppe für

Hämatologie und Onkologie (OSHO) am 5.11.2004 in Magdeburg vorgetragen.

Korrespondenzadresse

Prof. Dr. med. Karl Häußinger · Asklepios Fachkliniken München-Gauting •

Robert Koch-Allee 2 · 82131 Gauting · E-mail: k.haeussinger@asklepios.com

Eingang: 28. März 2005 • Nach Revision alkzeptiert: 29. Juli 2005

Bibliografie

Pneumologie 2005; 59: 588-591 @ Georg Thieme Verlag KG Stuttgart · New York

DOI $10.1055 / \mathrm{s}-2005-870987$

ISSN 0934-8387 
Hintergrund

Extramedulläre Manifestationen (EML) akuter myeloischer Leukämien (AML) werden initial häufig als Neoplasien wie Lymphome, Sarkome oder Karzinome fehlgedeutet [1]. Lokalisationen sind u.a. lymphoretikuläre Gewebe, Nervensystem, Haut, Schleimhäute und Lunge $[1,2]$. Pulmonale Infiltrate werden oft primär als infektiös gewertet. Zeitlich gesehen treten sie entweder synchron mit den initialen Blutbildveränderungen oder im Krankheitsverlauf, selten auch isoliert als primäre EML auf [1]. Bei der AML mit extramedullärer Manifestation findet sich meist eine myelomonozytäre oder monozytäre Differenzierung [1]. Der Verlauf von Fällen mit pulmonalen EML ist schlecht [3]. Bei AML mit pulmonaler Manifestation wurden bisher ausschließlich Fälle mit unzureichendem Therapieansprechen oder Frühletalität beschrieben [3-6].

Wir berichten über eine Patientin mit initial günstigem Verlauf einer AML mit symptomatischer, extramedullär-pulmonaler Manifestation.

\section{Kasuistik}

Eine 57-jährige Patientin wurde uns wegen einer therapieresistenten Pneumonie und mit dem dringenden Verdacht auf eine akute Leukämie aus einer auswärtigen Klinik zugewiesen. Seit Wochen bestanden Leistungsschwäche, Luftnot, Fieberschübe und Nachtschweiß. Bekannt waren eine Lippen-Kiefer-Gaumenspalte, arterielle Hypertonie, Linksherzinsuffizienz und ein insulinpflichtiger Diabetes mellitus Typ 2.

Die Patientin war in einem deutlich reduzierten Allgemein- und guten Ernährungszustand. Über beiden Lungen waren eine basale Klopfschalldämpfung und inspiratorisch trockene Rasselgeräusche ubiquitär zu verzeichnen. Es fand sich eine Anämie

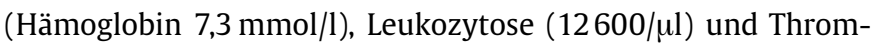
bozytopenie $(79000 / \mu \mathrm{l})$. Im Differentialblutbild sahen wir $68 \%$ Myeloblasten. Anhand der Knochenmarkzytologie (mittelgroße Blasten mit Kernfurchung und leicht granuliertem Zytoplasma), Zytochemie sowie Immunphänotypisierung (Blasten mit Expression von CD13/14/16/33/38/64 und HLA-DR; Koexpression von CD56 und schwach CD117, nicht jedoch CD34; Myeloperoxidase und Lactoferrin negativ) konnte die Diagnose einer akuten monoblastären Leukämie (nach WHO; M5a nach FAB) gestellt werden. In der Röntgenaufnahme des Thorax fielen ausgedehnte Verschattungen der Lungen auf (Abb. 1). Zur weiteren Abklärung des als therapierefraktäre Pneumonie interpretierten Befundes führten wir eine Bronchoskopie mit anschließender bronchoalveolärer Lavage (BAL) durch. Bronchoskopisch fanden sich keine Hinweise für eine Infektion; ebenfalls waren keine direkten oder indirekten Tumorzeichen nachweisbar. In der BAL-Flüssigkeit des Mittellappens (Spülung mit $60 \mathrm{ml}$ physiologischer Kochsalzlösung, Rückgewinnung von $35 \mathrm{ml}$ ) fanden sich zytologisch fast ausschließlich leukämische Blasten (Abb. 2). Eine Computertomographie (CT) des Thorax zeigte konfluierende, fleckförmige Verschattungen der Lungen (Abb. 3) sowie vergrößerte axilläre und mediastinale Lymphknoten. Es lag somit eine AML mit pulmonaler Manifestation vor. Die periphere Sauerstoffsättigung war unter $41 \mathrm{O}_{2} /$ min mit $91 \%$ erniedrigt. Die Blutgasanalyse er- brachte eine respiratorische Partialinsuffizienz $\left(\mathrm{pO}_{2} 53 \mathrm{~mm} \mathrm{Hg}\right.$, $\mathrm{pCO}_{2} 32 \mathrm{~mm} \mathrm{Hg}$ ); eine weiterführende Lungenfunktionsdiagnostik konnte aufgrund des schlechten Allgemeinzustandes primär nicht durchgeführt werden.

Nach Sicherung der Leukämiediagnose begannen wir mit einer Induktionschemotherapie bestehend aus niedrigdosiertem Cytarabin $\left(100 \mathrm{mg} / \mathrm{m}^{2}, \mathrm{~d} 1-7\right)$ und Idarubicin (12 mg/m², d3 - 5) [7]. Zur Eindämmung einer inflammatorischen Reaktion, und damit möglichen Verhinderung einer Lungenfibrose, verabfolgten wir zusätzlich Prednisolon ( $100 \mathrm{mg} / \mathrm{d}$ für 5 Tage, danach Ausschleichen über 30 Tage). Schon in den ersten Tagen nach Therapiebeginn verbesserte sich der Allgemeinzustand und vor allem die Dyspnoe zusehends. Im weiteren Verlauf führten wir Röntgenkontrollen (Abb.4, nach drei Monaten) und eine erneute CT durch, welche eine deutliche Verbesserung des pulmonalen Befundes und einen Rückgang der Lymphknotenschwellungen zeigten. Die Knochenmarkpunktion nach der Induktionstherapie erbrachte eine komplette hämatologische Remission (CR) der AML. Die Lungenfunktionsprüfung nach drei Monaten ergab noch eine Restriktion (VC 49\%, $\mathrm{FEV}_{1} 78 \%$ der Norm) und deutliche Störung der Diffusionskapazität ( $\mathrm{DL}_{\mathrm{CO}} 51 \%$ der Norm). Wir führten zwei Konsolidierungstherapien durch (zunächst mit Cytarabin [ $\left.100 \mathrm{mg} / \mathrm{m}^{2}, \mathrm{~d} 1-7\right]$ und Idarubicin [ $12 \mathrm{mg} / \mathrm{m}^{2}, \mathrm{~d} 1-3$ ], sodann mit mittelhochdosiertem Cytarabin $\left[2 \times 1000 \mathrm{mg} / \mathrm{m}^{2}\right.$, $\mathrm{d} 1,3,5]$ und Mitoxantron [ $\left.\left.10 \mathrm{mg} / \mathrm{m}^{2}, \mathrm{~d} 1-2\right]\right)$. Kurz vor der geplanten letzten Konsolidierungstherapie wurde die Patientin im Heimatkrankenhaus unter dem klinischen Bild einer therapierefraktären Pneumonie behandelt und verstarb leider dort innerhalb weniger Tage und insgesamt vier Monate nach Erreichen der CR. Eine Obduktion wurde abgelehnt.

\section{Diskussion}

EML finden sich im Sektionsgut in 90\% der Leukämiefälle; speziell leukämische Infiltrationen der Lunge bei AML werden in $28 \%$ beschrieben [2]. Klinisch wird eine pulmonale Manifestation in $20 \%$ der Fälle mit AML-M5 diagnostiziert [3]. Pulmonale EML treten eher bei peripheren Blastenwerten von mehr als $40 \%$ und Leukozytose auf $[3,8]$. Da leukämische Infiltrationen der Lungen jedoch auch bei aleukämischem Blutbild auftreten können, muss neben einer Leukostase auch eine aktive Adhäsion und Invasion leukämischer Blasten diskutiert werden. Diese Vorgänge werden durch zelluläre Adhäsionsmoleküle vermittelt und möglicherweise durch Zytokine induziert $[9,10]$. Extermann u. Mitarb. fanden unter 98 Patienten mit AML signifikant erhöhte Serumkonzentrationen von löslichem L-Selektin (sL-Selektin, CD62L) im Falle einer extramedullären Manifestation (46 von 98 Patienten). Lösliches L-Selektin (von sL-Selektin ${ }^{+}$-Blasten abgeschieden) inhibiert die u.a. von zellgebundenem L-Selektin abhängige Endothelzelladhäsion leukämischer Blasten. Diese Blasten können so über noch näher zu charakterisierende Mechanismen in extramedulläre Gewebe immigrieren [9].

Interessanterweise kommen EML - so wie in unserem Fall - in bis zu $40 \%$ bei Koexpression von CD56 vor $[1,11]$. In vitro konnte ein homophiler CD56-CD56-Bindungsmechanismus beschrieben werden [12]. CD56+-Blasten können so an CD56+-Zellen binden und in entsprechenden Geweben proliferieren [11]. 


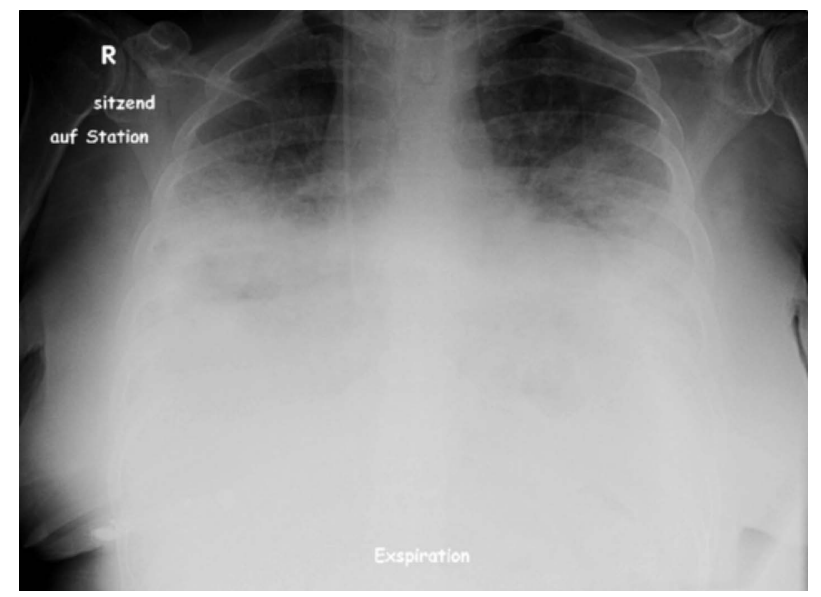

Abb. 1 Röntgenaufnahme des Thorax vor Therapie. Ausgedehnte Verschattungen beider Lungen.

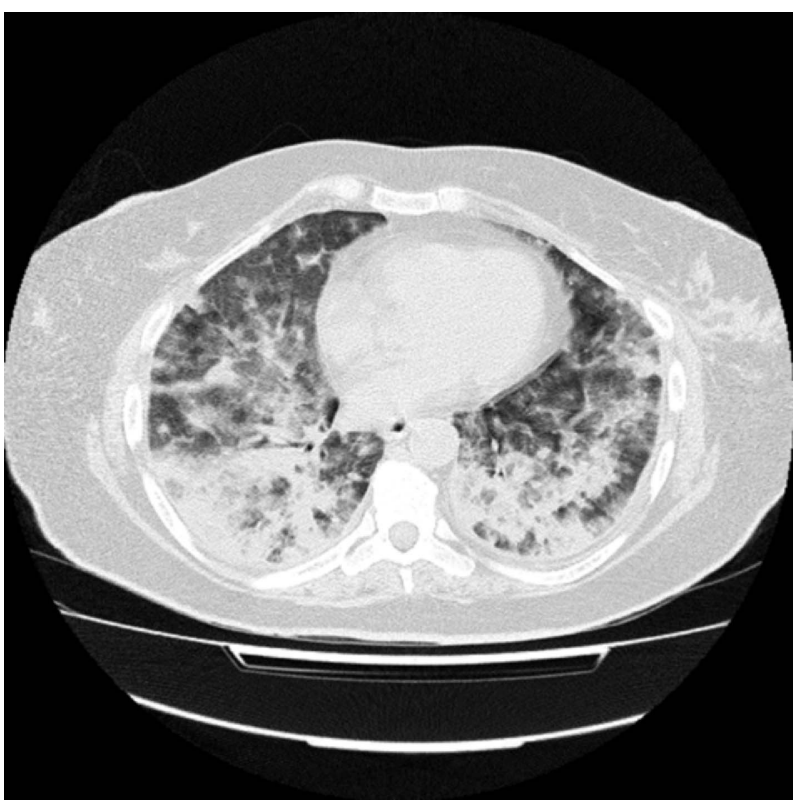

Abb. 3 CT des Thorax vor Therapie. Konfluierende, fleckförmige Verschattungen beider Lungen.

Zum Nachweis pulmonal-neoplastischer Infiltrationen bei Malignomen wird neben einer BAL auch eine transbronchiale Lungenbiopsie (TBLB) empfohlen. So konnte gezeigt werden, dass die Wahrscheinlichkeit der korrekten Diagnose bei der Kombination BAL und TBLB signifikant höher ist als bei alleiniger BAL [13]. In unserem Fall war die BAL jedoch bereits ausreichend. Bei hämatologischen Malignomen und unklarer Infiltration der Lunge handelt es sich in 8,5\% der Fälle um neoplastische und in der Mehrzahl (24\%) um infektiöse Infiltrationen; in 55\% der Fälle bleibt die Ätiologie unklar [13]. In Fällen von AML und unklaren pulmonalen Infiltraten ist in $60 \%$ ein diffuses Bild im Lungenröntgen zu verzeichnen [13]. Bei AML mit gesicherter pulmonaler Beteiligung handelt es sich in den meisten Fällen um alveoläre Infiltrationen; interstitielle Veränderungen werden weniger häufig beschrieben (bis zu 25\%) [3,14].

Der Verlauf der AML-M5 mit pulmonaler Manifestation ist schlecht: Aus Daten von Azoulay u. Mitarb. [3] geht hervor, dass

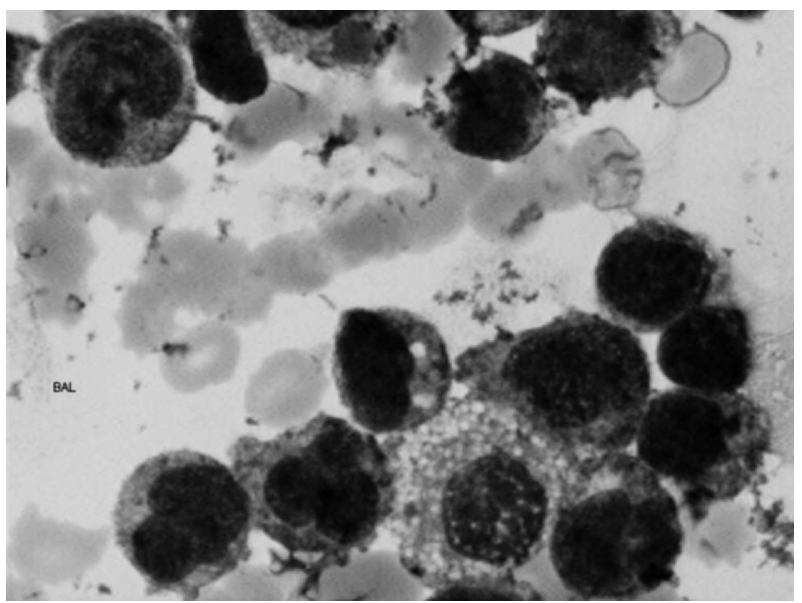

Abb. 2 Zytologie der BAL. Monozytäre Blasten (1000x).

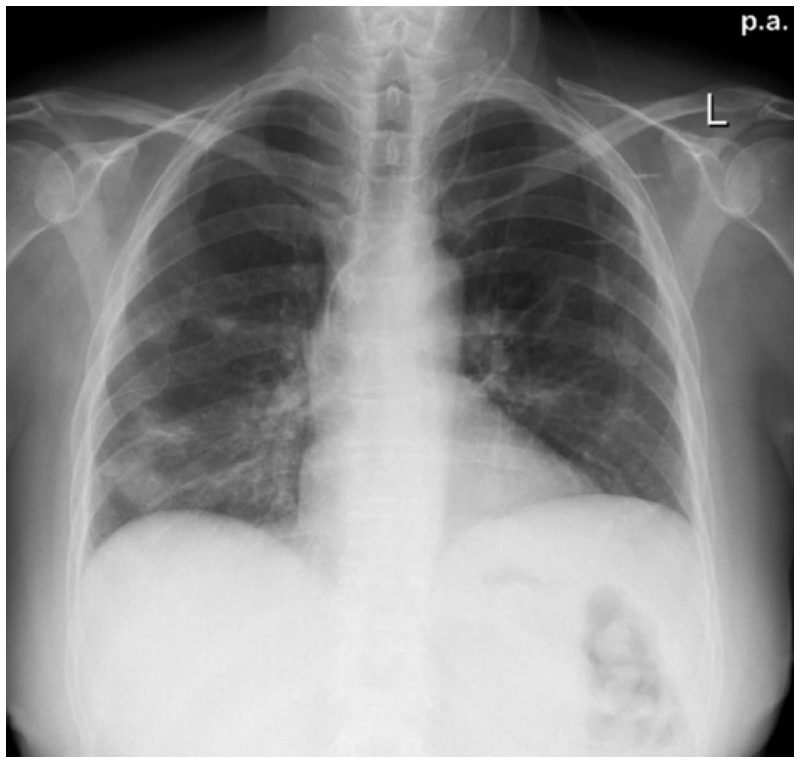

Abb. 4 Röntgenaufnahme des Thorax vor der zweiten Konsolidierungstherapie. Deutliche Rückbildung der pulmonalen Infiltrate/Verdichtungen (mit noch narbigen Resten).

alle 20 untersuchten Patienten mit AML-M5 und pulmonaler Beteiligung intensivmedizinisch betreut werden mussten und sich alle im Verlauf respiratorisch verschlechterten (Lyse-Pneumopathie); $75 \%$ dieser Patienten wurden beatmet und $50 \%$ verstarben während der Induktionstherapie. Die von uns angewandte zusätzliche Applikation von Prednisolon hat möglicherweise dazu beigetragen, die kritische pulmonale Situation der Patientin in soweit zu verbessern, dass eine Respiratortherapie vermieden und die Chemotherapie toleriert werden konnte. Es muss spekulativ bleiben, ob der letztlich doch letale Verlauf durch eine dauerhafte oder erneut eingesetzte Steroid-Medikation hätte verhindert werden können. Weiterhin wurde in unserem Fall nicht geklärt, ob sich hinter den residuellen pulmonalen Veränderungen (nach Chemotherapie) leukämische Infiltrate verbergen, oder ob es sich um narbiges Gewebe handelt. 


\section{Schlussfolgerungen}

Eine pulmonale Manifestation muss in differenzialdiagnostische Überlegungen bei Leukämie und unklaren Lungenbefunden einbezogen werden. Bei therapieresistenter Pneumonie sollte eine Bronchoskopie (mit BAL und ggf. TBLB) zur Diagnosesicherung durchgeführt werden. Das - im Vergleich zur Literatur - gute Therapieansprechen bzw. der initial gute Verlauf unseres Falles ist möglicherweise auf den Zusatz von Prednisolon zur antileukämischen Therapie zurückzuführen. Diese Einzelbeobachtung sollte klinisch systematisch untersucht werden.

\section{Literatur}

${ }^{1}$ Byrd JC, Edenfield WJ, Shields DJ et al. Extramedullary myeloid cell tumors in acute nonlymphocytic leukemia: a clinical review. J Clin Oncol 1995; 13: $1800-1816$

2 Barcos M, Lane W, Gomez GA et al. An autopsy study of 1206 acute and chronic leukemias (1958 to 1982). Cancer 1987; 60: 827-837

${ }^{3}$ Azoulay E, Fieux F, Moreau D et al. Acute monocytic leukemia presenting as acute respiratory failure. Am J Respir Crit Care Med 2003; 167: $1329-1333$

${ }^{4}$ Florschütz A, Schumann HJ, Erikson C et al. Primär pulmonale Manifestation einer extramedullären akuten myeloischen Leukämie. Pneumologie 2001; 55: $302-305$

${ }^{5}$ Meidenbauer N, Schlake G, Bross K et al. Symptomatische leukämische Infiltration der Lunge als Komplikation einer akuten myeloischen Leukämie. Dtsch Med Wschr 1998; 123: 110-113

${ }^{6}$ de Paz R, Canales MA, Hernandez-Navarro F. Granulocytic sarcoma (chloroma) of the lung. Br J Haematol 2003; 120: 176

7 Vogler WR, Velez-Garcia E, Weiner RS et al. A phase III trial comparing idarubicin and daunorubicin in combination with cytarabine in acute myelogenous leukemia: a Southeastern Cancer Study Group Study. J Clin Oncol 1992; 10: 1103 - 1111

${ }^{8}$ Kovalski R, Hansen-Flaschen J, Lodato RF et al. Localized leukemic pulmonary infiltrates. Diagnosis by bronchoscopy and resolution with therapy. Chest 1990; 97: 674-678

${ }^{9}$ Extermann M, Bacchi M, Monai $\mathrm{N}$ et al. Relationship between cleaved L-selectin levels and the outcome of acute myeloid leukemia. Blood 1998; 92: 3115-3122

${ }^{10}$ Stucki A, Rivier AS, Gikic M et al. Endothelial cell activation by myeloblasts: molecular mechanisms of leukostasis and leukemic cell dissemination. Blood 2001; 97: 2121 - 2129

${ }^{11}$ Iizuka Y, Aiso M, Oshimi $\mathrm{K}$ et al. Myeloblastoma formation in acute myeloid leukemia. Leuk Res 1992; 16: 665-671

12 Johnson CP, Fujimoto I, Perrin-Tricaud C et al. Mechanism of homophilic adhesion by the neural cell adhesion molecule: use of multiple domains and flexibility. Proc Natl Acad Sci USA 2004; 101: 6963-6968

${ }^{13}$ Mulabecirovic A, Gaulhofer P, Auner HW et al. Pulmonary infiltrates in patients with haematologic malignancies: transbronchial lung biopsy increases the diagnostic yield with respect to neoplastic infiltrates and toxic pneumonitis. Ann Hematol 2004; 83: 420-422

${ }^{14}$ Potenza L, Luppi M, Morselli M et al. Leukaemic pulmonary infiltrates in adult actute myeloid leukaemia: a high-resolution computerized tomography study. Br J Haematol 2003; 120: 1058 - 1061

\section{Erratum}

\section{K. Häußinger, M. Kohlhäufl, Epidemiologie und Diagnostik des Lungenkarzinoms - Teil 1. Pneumologie 2005; 59: 534 - 548.}

In Tab. $\mathbf{4}$ befand sich ein Fehler. Nachstehend die korrekte Tabelle.

Tab. 4 Prognose in Abhängigkeit vom Stadium nichtkleinzelliges Lungenkarzinom

\begin{tabular}{|c|c|}
\hline Stadium & 5 JÜR \% \\
\hline I A* T1N0M0 & 67 \\
\hline $\mathrm{IB}^{*} \mathrm{~T} 2 \mathrm{~N} 0 \mathrm{M} 0$ & 57 \\
\hline II $A^{*} \quad \mathrm{~T} 1 \mathrm{~N} 1 \mathrm{M} 0$ & 55 \\
\hline II B* T2N1M0 bis T3N0M0 & $38-39$ \\
\hline III A* T3N1M0 bis T1-3N2M0 & $23-25$ \\
\hline IV ${ }^{* *}$ jedes $\mathrm{T}$, jedes $\mathrm{N}, \mathrm{M} 1$ & 1 \\
\hline
\end{tabular}

*chirurgisches Staging

**klinisches Staging 